\title{
Mechanisms of autophagy regulation by adiponectin
}

\author{
Chan Rong ${ }^{\mathrm{a}}, \mathrm{Xin} \mathrm{Xu}^{\mathrm{a}, *}$ \\ ${ }^{a}$ Department of Geriatrics, Guangzhou First People's Hospital, Guangzhou, Guangdong Province, 51000, China.
}

\begin{abstract}
Adiponectin is a multifunctional adipocytokine produced predominantly by adipocytes, with potent antiinflammatory, insulin-sensitizing, and cytoprotective properties. Autophagy is a lysosome-dependent self-degradative process that mediates the degradation of damaged organelles, invading pathogens and protein aggregates, thus maintaining cellular homeostasis. Adiponectin performs different biological functions by regulating autophagy. This review attempts to elucidate the biological responses and potential mechanisms underlying adiponectin-induced autophagy, with an aim to guide the identification of new therapeutic targets of related diseases.
\end{abstract}

Keywords: Adiponectin, adiponectin receptors, autophagy

\section{Introduction}

Adiponectin is a healthy adipokine with positive metabolic effects, such as insulin-sensitizing, anti-inflammatory, antitumor, and cardiovascular protective functions. Adiponectin and its receptors play a vital role in regulating autophagy.

Autophagy is a highly conserved lysosome-dependent self-degradation process that mediates the degradation of damaged organelles, invading pathogens, and protein aggregates, thus maintaining cellular homeostasis. Autophagy dysregulation occurs in various pathologic conditions, including cancer, neurodegenerative disorders, and metabolic diseases [1]. A recent study indicated that inhibition of autophagy in mice through knockdown of autophagyassociated proteins significantly reduced the degradation of lipid droplets in brown adipose tissue [2]. Another study demonstrated that adiponectin induces autophagic flux in skeletal muscle cells and diminishes insulin resistance by alleviating ER stress, indicating that adiponectin mediates antidiabetic effects in an autophagy-dependent manner [3]. Furthermore, globular adiponectin induces Beclin-1 phosphorylation, inhibiting Beclin-1/Bcl-2 combination and mediates the induction of autophagy in macrophages

* Correspondence to: Xin Xu

Mailing address: Department of Geriatrics, Guangzhou First People's Hospital, Guangzhou, Guangdong Province, 51000, China.

Email: eyxuxin@scut.edu.cn

Received: 29 November 2021 / Accepted: 20 December 2021
[4]. In this review, we describe the function of adiponectin and its receptors in the process of autophagy and the underlying molecular signaling pathways.

We hope to provide a better understanding of the interrelation between adiponectin and autophagy, which may provide novel therapeutic directions for the treatment of related diseases.

\section{Adiponectin and adiponectin receptors}

Adiponectin, also known as AdipoQ, apM1, and GBP28, is primarily secreted by white adipose tissue [5]. It is a 30-kDa complement C1q-related protein with a globular $\mathrm{C}$-terminal domain and a collagenous $\mathrm{N}$-terminal domain, which usually circulates in oligomeric complexes as trimers, hexamers, and multimers [5, 6]. It regulates multiple molecular and cellular events, including maintaining energy homeostasis, lipid metabolism, insulin sensitivity, immune response, and inflammation [7].

Adiponectin carries out its diversified functions through two widely expressed receptors, AdipoR1 and AdipoR2, which are found in skeletal muscle, liver, and endothelial cells [8]. AdipoRon is an orally active adiponectinreceptor agonist; it can bind to AdipoR1 and AdipoR2 and activate AMP-activated protein kinase (AMPK) [9]. The function of these receptors varies depending on the target tissue.

\section{Adiponectin and autophagy induction}

Autophagy plays a critical role in maintaining cellular homeostasis and easing intracellular stress, including in- 
flammation response, oxidative stress, and endoplasmic reticulum stress. Impairment of autophagy may exacerbate diabetes-related metabolic disorders in insulin target tissues, including the liver, adipose tissue, skeletal muscle, and pancreatic $\beta$-cells $[10,11]$. Adiponectin plays a critical role in autophagy regulation in various types of cells or tissue, thus exerting different biological effects. It inhibits high-glucose-induced angiogenesis of RF/6A cells by suppressing autophagy [12]. Thus, autophagy may be one of the key mechanisms through which adiponectin and its receptors regulate various biological responses.

\section{Effects of adiponectin on inflammation modu- lation during autophagy induction}

Adipose tissue secretes adipokines and hormones, many of which are involved in inflammation, glucose homeostasis, and lipid metabolism. Autophagic flux is an important mechanism for various beneficial biological responses by adiponectin [13]. For example, autophagy affects lipid metabolism in adipose tissue and regulates cellular energy and nutrient storage [2]. Furthermore, the antiinflammatory effects of globular adiponectin may be mediated through autophagy induction. Globular adiponectin suppresses lipopolysaccharide-primed inflammasome activation and generation of active IL- $1 \beta$ in murine peritoneal macrophages by upregulating autophagy and active AMPK signaling [14]. On the contrary, inhibition of autophagy in adipocytes was associated with a significant upregulation of adiponectin expression and a decrease in proinflammatory markers [15]. Defects of autophagy-related genes such as Atg3 and Atg16L1 in fully differentiated adipocytes cause inflammation, insulin resistance, and mitochondrial dysfunction. Moreover, Atg3 and Atg16L1 are required for proper mitochondrial function in mature adipocytes, yet postdevelopmental ablation of autophagy causes peripheral insulin resistance regardless of diet or adiposity [16]. This evidence indicates that autophagy is critical for lipid accumulation and adipocyte differentiation factors [17].

Adiponectin is generally considered to possess cytoprotective properties. For example, adipocyte-specific gene Atg5 knockout mice had increased circulating levels of adiponectin and protected against alcohol-induced adipose atrophy and liver injury [18]. Moreover, adiponectin has cytoprotective and antiinflammation properties by inhibiting autophagy. An in vitro study showed that globular adiponectin contributes to Beclin-1 phosphorylation and Bcl-2 mRNA destabilization in macrophages and exerts an anti-inflammatory effect. The interaction between Beclin-1 and Bcl-2 is regarded as a critical step in the regulation of autophagy induction; inhibition of such an interaction is a plausible mechanism for the initiation of autophagy. Consistently, another study observed that adiponectin caused Bcl-2 mRNA destabilization and consequently activated autophagy in macrophages [19]. A study further determined the effects of globular adiponectin on Beclin-1 phosphorylation and Bcl-2 mRNA stability, and investi- gated their role in suppressing inflammatory mediators. Interestingly, globular adiponectin was found to suppress the inflammation response by inhibiting the formation of Beclin-1 and Bcl-2 complexes and inducing macrophage autophagy [4]. Adiponectin exhibits protective effects against hepatotoxicity; ER stress leads to inflammasome activation in hepatocytes. For instance, globular adiponectin significantly suppressed expression of ER stress marker genes and promoted inflammasome activation, hence protecting hepatocytes against cell death by autophagy induction, indicating that adiponectin possesses hepatocyte protection, at least in part, via autophagy induction [20]. Adiponectin potently suppresses the production of inflammatory mediators and promotes autophagy by inhibiting inflammatory responses, thus exhibiting cytoprotective properties. Further research should explore the effects of adiponectin on inflammasomes, which can help in the treatment of inflammatory-related diseases.

\section{Effects of adiponectin on autophagy induction by regulating the AMPK signaling pathway}

AMPK is a central regulator of energy homeostasis. In many cases, adiponectin-induced autophagy is related to the AMPK signaling pathway [21]. Autophagy dysregulation is responsible for various diseases, including type 2 diabetes, myocardial injury, and renal damage; deficiency in autophagy is associated with metabolic disorders [22, 23]. Otherwise, regulated autophagy is a critical component of a healthy skeletal muscle mass [24].

Cardiomyocyte autophagy is vital for maintaining cardiac function. Decreased myocardial autophagic flux results in cardiac dysfunction and cardiomyocyte death. By contrast, it activates autophagy by globular adiponectin in myoblasts and promotes myoblast survival and apoptosis via an AMPK-dependent mechanism [25]. A study confirmed that adiponectin increases autophagic flux through promotion of AMPK phosphorylation. Adiponectin deficiency could aggravate the downregulation of myocardial AMPK phosphorylation, autophagic flux, and cardiac function. By contrast, exogenous administration of adiponectin reverses the decline of AMPK phosphorylation and autophagic flux and eventually reduce cardiomyocyte death [26]. AdipoRon, as an adiponectin receptor agonist, inhibits myeloma cell proliferation and induces apoptosis, and AMPK/autophagy pathway may be one of its mechanisms [9]. Moreover, AdipoRon is a cardioprotective molecule; deficiency of ADIPOQ markedly increases myocardial ischemia-reperfusion (MI-R) injury. Hypoadiponectinemia in a diabetic model impairs autophagic flux, and consequently enhances MI-R injury. Additionally, adipoR activation restores AMPK-mediated autophagosome formation and antioxidant-mediated autophagosome clearance, manifesting a novel intervention effective against MI-R injury in diabetic conditions [27]. In vitro studies have demonstrated that AdipoRon promotes autophagic flux 
through activation of AMPK/ULK1 pathway, thus inhibiting renal fibrosis [28]. Adipose tissue specifically secretes autophagy protein Becn1, and then facilitates the secretion of adiponectin [29]. Furthermore, Becn1 regulates AMPK activity and improves insulin sensitivity by promoting adiponectin secretion [30]. In addition, adiponectin knockout (Ad-KO) in mice induces insulin resistance and autophagy, and exogenous adiponectin unregulates the expression of autophagy-related genes LC3-II and Beclin1. In vitro, adiponectin enhanced autophagic flux in cultured muscle cells in an AMPK-dependent manner. Taken together, the study demonstrated that adiponectin stimulated skeletal muscle autophagy and alleviated HFD-induced insulin resistance and metabolic dysfunction in skeletal muscle [31]. Moreover, adipoRon upregulated LC3-II/ LC3-I level, downregulated p62 protein level in multiple myeloma cells, and significantly inhibited MM cell proliferation and increased their expression of apoptosis-related proteins. Moreover, AdipoRon upregulated p-AMPK and its downstream p-ACC in MPC-11. Globular adiponectin induces autophagy in chondrocytes by increasing the formation of Beclin-1 and LC3B and P62 degradation, thus exhibiting an antiapoptotic effect, indicating that global adiponectin possesses antiapoptotic properties by inducing autophagy. In this study, the authors demonstrated that global adiponectin induced a high expression of $\mathrm{p}$ AMPK accompanying a high expression of Beclin-1 and LC3-II in chondrocytes. Furthermore, the application of the inhibitor of AMPK significantly blocked the global adiponectin-induced autophagy in chondrocytes [32].

To investigate the therapeutic value of AdipoRon, an adiponectin receptor agonist, and the molecular mechanism on chondrocyte calcification, an in vitro study investigating the effect on autophagy by AdipoRon in chondrocytes revealed that adiponRon decreased calcification and ALP activity by promoting autophagy. However, these activities were blocked by the AMPK inhibitor [33]. Epidemiological studies have revealed that patients with breast cancer generally have low levels of circulating AdipoQ, which indicates a poor prognosis. Moreover, elevated expression of AdipoQ in breast tissue is closely associated with advanced stages of the disease. Globular adiponectin upregulated microtubule-associated protein 1 light chain 3 beta (LC3B)-II and intracellular LC3B puncta, which are indicators of autophagosome formation, suggesting that adiponectin contributes to autophagic induction, hence promoting the migration and invasion abilities of breast cancer cells [34]. Consistently, Chung et al. reported that ADIPOQ/adiponectin induces accumulation of autophagosomes in breast cancer cells, inhibiting breast cancer growth and inducing apoptosis. Otherwise, AMPK-inhibition abrogates ADIPOQ/adiponectin-induced ULK1-activation, LC3B-turnover, and p62-degradation, yet AMPK activation reverses those effects [21]. These data indicate that ADIPOQ/adiponectin induces autophagy in breast cancer cells through the modulation of the AMPK-ULK1 axis. Collectively, the results suggest that by adiponectin modulation autophagy via the AMPK signaling pathway.

\section{Effects of adiponectin on autophagy induction by regulating oxidative stress and ER stress}

Adiponectin is reported to possess cardioprotective properties, and studies have shown that the mechanism may be related to modulation of mitochondrial function or ER stress. ER stress promotes cardiac lipotoxicity in cardiac myocytes. Globular adiponectin (gAcrp) significantly upregulates the expression of various ER stress markers in macrophages, and inhibition of ER stress prominently suppresses gAcrp-induced autophagy [35]. Pretreatment with exogenous APN inhibited ER stress and activated autophagy, thereby protecting cardiomyocytes against apoptosis through AMPK activation [36]. Similarly, another study showed that exogenous administration of adiponectin significantly reduced oxidative stress and increased the expression of anti-oxidative enzymes, resulting in autophagy stimulation, hence inhibiting apoptosis and diminishing brain tissue injury [37].

Globular CTRP9 (gCTRP9), a newly identified adiponectin paralog, increases the ratio of LC3II/I and upregulate autophagy-related gene ATG5, and decrease the level of $\mathrm{P} 62$, which is vital to autophagosome formation. Moreover, gCTRP9 restored the loss of mitochondrial membrane potential, suppressed ROS generation, and reduced myocyte death. These results suggest that adiponectin paralog protects against oxidative stress-mediated damage in cardiomyocytes by enhancing autophagy [38]. Shi et al. demonstrated that in the testes of diabetic mice, globular adiponectin upregulated the expression of autophagyrelated protein while inhibiting the expression of NAD(P) $\mathrm{H}$-quinone oxidoreductase 1 , heme oxygenase-1, and superoxide dismutase, suggesting that globular adiponectin exhibits a protective effect on diabetic mice by inhibiting oxidative stress and ER stress and inducing autophagy [39]. In conclusion, adiponectin exerts a cytoprotective effect likely by inducing autophagy through the modulation of oxidative stress and ER stress.

\section{Selective form of autophagy induced by adipo- nectin}

Adiponectin targets specific forms of autophagy: mitophagy, lipopagy, and endoplasmic reticulum autophagy (ER-phagy). Herein, we elucidate the association and the underlying mechanism involved as follows.

The elimination of damaged mitochondria is critical for ensuring energy supply and maintaining mitochondrial quality. Mitochondrial autophagy (mitophagy), characterized by selectively excluding damaged mitochondria via a specific autophagic pathway, is one of the catabolic processes by which dysfunctional mitochondria are degraded [40]. It is crucial for mitochondrial quality control and moderating mitochondrial homeostasis and plays a critical role in cytoprotection [41, 42]. Adiponectin modulates insulin responsiveness by regulating mitophagy and maintains mitochondrial homeostasis, but the underlying 
mechanisms remain unknown. It also promotes mitophagy and improves lung functional recovery in type 2 diabetic rats, suppressing oxidative damage, decreasing inflammation response, diminishing cell apoptosis, and preserving mitochondrial function [43]. Chronic intermittent hypoxia causes disturbances of genioglossal mitophagy, while supplementation of exogenous adiponectin alleviated the damage to mitochondrial structure and function by increasing mitophagy [44]. An in vitro study demonstrated that adiponectin suppressed the over-production of ROS by activating the Nrf2/HO-1 pathway, suppressed $\mathrm{H}_{2} \mathrm{O}_{2}$-induced mitophagy, and partially inhibited the colocalization of autophagosomes/lysosomes with mitochondria. Otherwise, adiponectin downregulates the expression of both $\mathrm{Bax}$ and $\mathrm{Bax} / \mathrm{Bcl}-2$ protein induced by oxidative stress [45]. These findings suggest that APN moderately regulates oxidative stress-induced mitophagy and suppresses apoptosis. Similarly, globular adiponectin upregulates mitophagy while reducing the rate of hepatocyte apoptosis induced by intermittent hypoxia [46]. In summary, adiponectin may protect against tissue damage by upregulating mitophagy, but the concrete underlying mechanisms remain unclear.

Lipophagy is another selective form of autophagy characterized by selective degradation of lipid droplets (LDs). It is beneficial in maintaining lipid homeostasis. Increased lipophagy is potent in decreasing abnormal lipid accumu- lation by removing ectopic LDs, thus alleviating insulin resistance and $\beta$-cell impairment [47]. Disturbances in lipophagy have been linked to Nonalcoholic fatty liver disease (NAFLD)and hepatic triglyceride accumulation, obesity, liver steatosis, and atherosclerosis [48, 49]. Similarly, in a recent study, the authors observed that lipophagy deficiency in the tubular cells of patients with diabetic nephropathy and $\mathrm{db} / \mathrm{db}$ mice was accompanied by significantly ectopic lipid deposition, oxidative stress, and apoptosis. Furthermore, AdipoRon administration moderated these damages by increasing lipophagy, and the effects were blocked partially by AdipoR1 siRNA, an autophagy inhibitor, and enhanced by AMPK activator [50]. The results indicated that AdipoRon can reduce intrarenal lipotoxicity-associated renal injury by upregulating lipophagy by activating the AdipoR1/AMPK pathway.

ER-phagy enables protein and lipid synthesis, ion homeostasis, and organelle communication via constant ER turnover and modulation [51]. Globular adiponectin upregulates ER-phagy to alleviate ER stress and attenuates H9C2 cardiomyocyte apoptosis induced by CIH through AMPK activation [52]. Interruption of the adiponectin signaling pathway mimics perturbed or inadequate nutrient intake, triggers catabolic processes such as selective forms of autophagy, including ER-phagy and lipophagy, to acquire and mobilize internal nutrient stores, enhances survival, and promotes longevity in C. elegans [53].

Table1. Effects of adiponectin on autophagy regulation.

\begin{tabular}{|c|c|c|}
\hline Experimental models & Biological responses & References \\
\hline L6 skeletal muscle cells & $\begin{array}{l}\text { Adiponectin-induced autophagy in skeletal muscle cells and alleviated ER stress and } \\
\text { insulin resistance. }\end{array}$ & Ahlstrom, P., et al. (2017) \\
\hline Breast cancer cells & $\begin{array}{l}\text { Adiponectin induces autophagic cell death in breast cancer, and inhibits breast cancer } \\
\text { growth and induces apoptosis. }\end{array}$ & Chung, S. J., et al. (2017) \\
\hline Chondrocytes & $\begin{array}{l}\text { Globular adiponectin protected chondrocytes by inducing autophagy via AMPK/mTOR } \\
\text { signal-pathway activation. } \\
\text { AdipoRon alleviates the calcification of chondrocytes via activating AMPK-mTOR } \\
\text { signaling to promote autophagy. }\end{array}$ & $\begin{array}{l}\text { Hu, J., et al. (2017) } \\
\text { Duan, Z. X., et al. (2020) }\end{array}$ \\
\hline Macrophages & $\begin{array}{l}\text { Adiponectin inhibits LPS-primed inflammasomes activation via autophagy induction and } \\
\text { AMPK signaling activation. } \\
\text { Gene silencing of p } 62 \text { prevented gAcrp-induced increases in autophagy-related genes and } \\
\text { autophagosome formation. } \\
\text { Enhanced interaction of Bcl-2 with Beclin-1 prevented gAcrp-induced autophagy } \\
\text { activation. }\end{array}$ & $\begin{array}{l}\text { Kim, M. J., et al. (2017) } \\
\text { Tilija Pun, N., et al. }(2017) \\
\text { Shrestha, A., et al. }(2018)\end{array}$ \\
\hline C2C12 myoblasts & $\begin{array}{l}\text { Adiponectin protected } \mathrm{C} 2 \mathrm{C} 12 \text { myoblasts against oxidative stress-induced apoptosis and } \\
\text { suppressed } \mathrm{H}(2) \mathrm{O}(2) \text {-induced mitophagy. }\end{array}$ & Ren, Y., et al. (2017) \\
\hline $\begin{array}{l}\text { Adipoq knockout } \\
(\operatorname{adipoq}(-/-)) \text { mice }\end{array}$ & $\begin{array}{l}\text { Hypoadiponectinemia impairs autophagic flux, contributing to enhanced MI-R injury in } \\
\text { the diabetic state. } \\
\text { gAcrp protects hepatocytes against cell death by modulating ER stress and inflammasome } \\
\text { activation via autophagy induction. } \\
\text { gAPN alleviated mitochondrial injury and hepatocyte apoptosis by upregulating Pink1/ } \\
\text { Parkin-mediated mitophagy. }\end{array}$ & $\begin{array}{l}\text { Wang, Y., et al. (2017) } \\
\text { Kim, E. H. and P. H. Park } \\
\text { (2018) } \\
\text { Ding, W., et al. (2021) }\end{array}$ \\
\hline hepatocyte & $\begin{array}{l}\text { APN inhibited ER stress and activated autophagy through AMPK activation, thus } \\
\text { alleviating HL-1 apoptosis. } \\
\text { gAPN upregulated ER-phagy to extenuate ER stress, and mitigated cardiomyocytes } \\
\text { apoptosis through AMPK activation. } \\
\text { gCTRP9 protects cardiomyocytes through enhanced autophagic flux. }\end{array}$ & $\begin{array}{l}\text { Li, B., et al. (2020) } \\
\text { Zhang, Q., et al. (2020) } \\
\text { Zuo, A., et al. (2020). }\end{array}$ \\
\hline Diabetic mice & $\begin{array}{l}\text { Adiponectin protects the testes of diabetic mice by inducing autophagy and inhibiting ER } \\
\text { stress and oxidative stress. } \\
\text { Adiponectin attenuated oxidative stress, inflammation, apoptosis and mitochondrial } \\
\text { dysfunction via activation of mitophagy in diabetic lung IR injury. }\end{array}$ & $\begin{array}{l}\text { Shi, W., et al. }(2020) \\
\text { Jiang, T., et al. }(2021)\end{array}$ \\
\hline
\end{tabular}




\section{Conclusions}

In summary, adiponectin is a healthy adipocytokine that has anti-inflammatory and antiapoptotic properties, favorable effects on intermediary metabolism, and cardiovascular, renal, and liver protection (Table 1). Adiponectin has multibiological effects on a wide variety of metabolic pathways, many of which are mediated via autophagy induction. Inflammation response, AMPK activation, oxidative and ER stress, and related signaling pathways are the main pathways by which adiponectin modulates autophagy. However, most current research has focused on molecular or cellular biological reactions. Future studies should elucidate the precise mechanisms by which adiponectin/ adipoRs regulate autophagy and its potential physiological functions, as well as evaluate autophagy regulation and implicated biological responses by adiponectin in vivo. A better understanding of the regulation of autophagy by adiponectin can guide preclinical and clinical studies in the treatment of metabolic diseases and cancer.

\section{Declarations}

Authors' contributions: The author contributed solely to the article.

Availability of data and materials: Not applicable.

Financial support and sponsorship: None.

Conflicts of interest: $\mathrm{Xin} \mathrm{Xu}$ is a member of the Editorial Board of Aging Pathobiology and Therapeutics. All authors declare no conflicts of interest and were not involved in the journal's review or desicions related to this manuscript.

Ethical approval and consent to participate: Not applicable.

\section{References}

1. Glick D, Barth S, Macleod K F. Autophagy: Cellular and molecular mechanisms. The Journal of Pathology, 2010, 221(1): 3-12.

2. Nakajima S, Nishimoto $Y$, Tateya $S$, et al. Fat-specific protein $27 \alpha$ inhibits autophagy-dependent lipid droplet breakdown in white adipocytes. Journal of Diabetes Investigation, 2019, 10(6): 1419-1429.

3. Ahlstrom P, Rai E, Chakma S, et al. Adiponectin improves insulin sensitivity via activation of autophagic flux. Journal of Molecular Endocrinology, 2017, 59(4): 339-350.

4. Tilija Pun N, Park P H. Adiponectin inhibits inflammatory cytokines production by Beclin-1 phosphorylation and B-cell lymphoma 2 mRNA destabilization: role for autophagy induction. British Journal of Pharmacology, 2018, 175(7): 1066-1084.

5. Nakano $\mathrm{Y}$, Tobe $\mathrm{T}$, Choi-Miura $\mathrm{N} \mathrm{H}$, et al. Isolation and characterization of GBP28, a novel gelatin-binding pro- tein purified from human plasma. The Journal of Biochemistry, 1996, 120(4): 803-812.

6. Davis K E, Scherer P E. Adiponectin: No longer the lone soul in the fight against insulin resistance? Biochemical Journal, 2008, 416(2): e7-e9.

7. Khoramipour K, Chamari K, Hekmatikar A A, et al. Adiponectin: Structure, physiological functions, role in diseases, and effects of nutrition. Nutrients, 2021, 13(4): 1180.

8. Cai J, Hu Q, Lin H, et al. Adiponectin/adiponectin receptors mRNA expression profiles in chickens and their response to feed restriction. Poultry Science, 2021, 100(12): 101480.

9. Wang S J, Wang C, Wang W Q, et al. Adiponectin receptor agonist adiporon inhibits the proliferation of myeloma cells via the AMPK/autophagy pathway. Zhongguo Shi Yan Xue Ye Xue Za Zhi, 2020, 28(1): 171-176.

10. Pearson G L, Gingerich M A, Walker E M, et al. A selective look at autophagy in pancreatic $\beta$-cells. Diabetes, 2021.

11. Fan Y, Lu J, Liu J, et al. 1, 3-dichloro-2-propanol induced hepatic lipid accumulation by inhibiting autophagy via AKT/mTOR/FOXO1 pathway in mice. Food and Chemical Toxicology, 2021, 157: 112578.

12. Li R, Du J, Yao Y, et al. Adiponectin inhibits high glucoseinduced angiogenesis via inhibiting autophagy in RF/6A cells. Journal of Cellular Physiology, 2019, 234(11): 20566-20576.

13. Pun N T, Park P H. Role of p62 in the suppression of inflammatory cytokine production by adiponectin in macrophages: Involvement of autophagy and p21/Nrf2 axis. Scientific Reports, 2017, 7(1): 1-17.

14. Kim M J, Kim E H, Pun N T J, et al. Globular adiponectin inhibits lipopolysaccharide-primed inflammasomes activation in macrophages via autophagy induction: the critical role of AMPK signaling. International journal of Molecular Sciences, 2017, 18(6): 1275.

15. Kosacka J, Nowicki M, Paeschke S, et al. Up-regulated autophagy: As a protective factor in adipose tissue of WOKW rats with metabolic syndrome. Diabetology \& Metabolic Syndrome, 2018, 10(1): 1-15.

16. Cai J, Pires K M, Ferhat M, et al. Autophagy ablation in adipocytes induces insulin resistance and reveals roles for lipid peroxide and Nrf2 signaling in adipose-liver crosstalk. Cell Reports, 2018, 25(7): 1708-1717.

17. Zhang $X$, Liu $Q$ Zhang $X$, et al. FOXO3a regulates lipid accumulation and adipocyte inflammation in adipocytes through autophagy. Inflammation Research, 2021, 70(5): 591-603.

18. Li Y, Chao X, Wang S, et al. Role of mechanistic target of rapamycin and autophagy in alcohol-induced adipose atrophy and liver injury. The American Journal of Pathology, 2020, 190(1): 158-175.

19. Shrestha A, Pun N T, Park P H. ZFP36L1 and AUF1 induction contribute to the suppression of inflammatory mediators expression by globular adiponectin via autophagy induction in macrophages. Biomolecules \& Therapeutics, 2018, 26(5): 446-457.

20. Kim E H, Park P H. Globular adiponectin protects rat 
hepatocytes against acetaminophen-induced cell death via modulation of the inflammasome activation and ER stress: critical role of autophagy induction. Biochemical Pharmacology, 2018, 154: 278-292.

21. Chung S J, Nagaraju G P, Nagalingam A, et al. ADIPOQ/adiponectin induces cytotoxic autophagy in breast cancer cells through STK11/LKB1-mediated activation of the AMPK-ULK1 axis. Autophagy, 2017, 13(8): 1386-1403.

22. Tao T, Xu H. Autophagy and obesity and diabetes. Springer, 2020: 445-461.

23. Kitada M, Koya D. Autophagy in metabolic disease and ageing. Nature Reviews Endocrinology, 2021, 17(11): 647-661.

24. Thapaliya S, Runkana A, McMullen M R, et al. Alcoholinduced autophagy contributes to loss in skeletal muscle mass. Autophagy, 2014, 10(4): 677-690.

25. Gamberi T, Modesti A, Magherini F, et al. Activation of autophagy by globular adiponectin is required for muscle differentiation. Biochimica et Biophysica Acta (BBA)Molecular Cell Research, 2016, 1863(4): 694-702.

26. Sun C, Lu J, Long Y, et al. Adiponectin up-regulates the decrease of myocardial autophagic flux induced by $\beta 1$ adrenergic receptor autoantibody partly dependent on AMPK. Journal of Cellular and Molecular Medicine, 2021, 25(17): 8464-8478.

27. Wang Y, Liang B, Lau W B, et al. Restoring diabetesinduced autophagic flux arrest in ischemic/reperfused heart by ADIPOR (adiponectin receptor) activation involves both AMPK-dependent and AMPK-independent signaling. Autophagy, 2017, 13(11): 1855-1869.

28. Li Y, Song B, Ruan C, et al. Adiporon attenuates hypertension-induced epithelial-mesenchymal transition and renal fibrosis via promoting epithelial autophagy. Journal of Cardiovascular Translational Research, 2020: 1-8.

29. Kuramoto $\mathrm{K}, \mathrm{He} \mathrm{C}$. The secretory function of BECN 1 in metabolic regulation. Autophagy, 2021, 17(10): 32623263.

30. Kuramoto K, Kim Y J, Hong J H, et al. The autophagy protein Becn 1 improves insulin sensitivity by promoting adiponectin secretion via exocyst binding. Cell Reports, 2021, 35(8): 109184.

31. Liu Y, Palanivel R, Rai E, et al. Adiponectin stimulates autophagy and reduces oxidative stress to enhance insulin sensitivity during high-fat diet feeding in mice. Diabetes, 2015, 64(1): 36-48.

32. Hu J, Cui W, Ding W, et al. Globular adiponectin attenuated $\mathrm{H}_{2} \mathrm{O}_{2}$-induced apoptosis in rat chondrocytes by inducing autophagy through the AMPK/mTOR pathway. Cellular Physiology and Biochemistry, 2017, 43(1): 367382.

33. Duan Z, Tu C, Liu Q, et al. Adiponectin receptor agonist adiporon attenuates calcification of osteoarthritis chondrocytes by promoting autophagy. Journal of Cellular Biochemistry, 2020, 121(5-6): 3333-3344.

34. Falk L E, Liu J, Li Y I, et al. Globular adiponectin enhances invasion in human breast cancer cells. Oncology Letters, 2016, 11(1): 633-641.

35. Oh H J, Lee S, Park P H. ER stress contributes to autoph- agy induction by adiponectin in macrophages: Implication in cell survival and suppression of inflammatory response. Cytokine, 2020, 127: 154959.

36. Li B, Zhang B, Liu N, et al. Adiponectin protects HL-1 cardiomyocytes against rotenone-induced cytotoxicity through AMPK activation. Toxicology Letters, 2020, 335: 82-90.

37. He Y, Liu B, Yao P, et al. Adiponectin inhibits cardiac arrest/cardiopulmonary resuscitation-induced apoptosis in brain by increasing autophagy involved in AdipoR1-AMPK signaling. Molecular Medicine Reports, 2020, 22(2): 870-878.

38. Zuo A, Li J, Zhao X, et al. Globular CTRP9 protects cardiomyocytes from palmitic acid-induced oxidative stress by enhancing autophagic flux. Chemico-biological Interactions, 2020, 329: 109094.

39. Shi W, Guo Z, Ji Y, et al. The protective effect of recombinant globular adiponectin on testis by modulating autophagy, endoplasmic reticulum stress and oxidative stress in streptozotocin-induced diabetic mice. European Journal of Pharmacology, 2020, 879: 173132.

40. Springer M Z, Macleod K F. In Brief: Mitophagy: mechanisms and role in human disease. 2016.

41. Davidson S M, Adameová A, Barile L, et al. Mitochondrial and mitochondrial-independent pathways of myocardial cell death during ischaemia and reperfusion injury. Journal of Cellular and Molecular Medicine, 2020, 24(7): 3795-3806.

42. Morales P E, Arias-Durán C, Ávalos-Guajardo Y, et al. Emerging role of mitophagy in cardiovascular physiology and pathology. Molecular Aspects of Medicine, 2020, 71: 100822.

43. Jiang T, Liu T, Deng X, et al. Adiponectin ameliorates lung ischemia-reperfusion injury through SIRT1-PINK1 signaling-mediated mitophagy in type 2 diabetic rats. Respiratory Research, 2021, 22(1): 1-19.

44. Wang W, Ding W, Huang H, et al. The role of mitophagy in the mechanism of genioglossal dysfunction caused by chronic intermittent hypoxia and the protective effect of adiponectin. Sleep and Breathing, 2020: 1-10.

45. Ren Y, Li Y, Yan J, et al. Adiponectin modulates oxidative stress-induced mitophagy and protects $\mathrm{C} 2 \mathrm{C} 12$ myoblasts against apoptosis. Scientific reports, 2017, 7(1): 1-12.

46. Ding W, Dong Y, Zhang X. Globular adiponectin protects hepatocytes against intermittent hypoxia-induced injury via Pink1/Parkin-mediated mitophagy induction. Sleep and Breathing, 2021: 1-9.

47. Huang M, Yang X, Wang Z, et al. Lipophagy: A new perspective of natural products in type 2 diabetes mellitus treatment. Diabetes, Metabolic Syndrome and Obesity: Targets and Therapy, 2021, 14: 2985.

48. Carotti S, Zalfa F, Ruggiero S, et al. Lipophagy impairment is associated with disease progression in NAFLD. Frontiers in Physiology, 2020, 11: 850.

49. Kloska A, Węsierska M, Malinowska M, et al. Lipophagy and lipolysis status in lipid storage and lipid metabolism diseases. International journal of Molecular Sciences, 2020, 21(17): 6113. 
50. Han Y, Xiong S, Zhao H, et al. Lipophagy deficiency exacerbates ectopic lipid accumulation and tubular cells injury in diabetic nephropathy. Cell Death \& Disease, 2021, 12(11): 1-13.

51. Khaminets A, Heinrich T, Mari M, et al. Regulation of endoplasmic reticulum turnover by selective autophagy. Nature, 2015, 522(7556): 354-358.

52. Zhang Q, Zhang X, Ding N, et al. Globular adiponectin alleviates chronic intermittent hypoxia-induced H9C2 cardiomyocytes apoptosis via ER-phagy induction. Cell Cycle, 2020, 19(22): 3140-3153.

53. Kyriakakis E, Charmpilas N, Tavernarakis N. Differential adiponectin signalling couples ER stress with lipid metabolism to modulate ageing in C. elegans. Scientific Reports, 2017, 7(1): 1-13.

Cite this article as: Rong $\mathrm{C}, \mathrm{Xu} \mathrm{X}$. Mechanisms of autophagy regulation by adiponectin[J]. Aging Pathobiology and Therapeutics, 2021, 3(4): 95-101. 